\title{
Comparison of ATP sulfurylase 2 from selenium hyperaccumulator Stanleya pinnata and non-accumulator Stanleya elata reveals differential intracellular localization and enzyme activity levels ${ }^{\text {i⿱ }}$
}

\author{
Ying Jiang ${ }^{\mathrm{a}, \mathrm{b}}$, Michela Schiavon ${ }^{\mathrm{b}, \mathrm{c}}$, Leonardo W. Lima ${ }^{\mathrm{b}}$, Tripti ${ }^{\mathrm{b}, \mathrm{d}}$, Rachel R. Jones ${ }^{\mathrm{b}}$, \\ Ali F. El Mehdawi ${ }^{\mathrm{b}}$, Suzanne Royer ${ }^{\mathrm{b}}$, Zhaohai Zeng ${ }^{\mathrm{a}}$, Yuegao Hu ${ }^{\mathrm{a}}$, Elizabeth A.H. Pilon-Smits ${ }^{\mathrm{b}}$, \\ Marinus Pilon ${ }^{\mathrm{b}, *}$ \\ ${ }^{\text {a }}$ College of Agronomy and Biotechnology, China Agricultural University, Beijing 100193, China \\ b Department of Biology, Colorado State University, Fort Collins, CO 80523, USA \\ ${ }^{\mathrm{c}}$ DAFNAE Department, Padova University, Agripolis, 35020, Legnaro, Padua, Italy \\ d Department of Experimental Biology and Biotechnology, Institute of Natural Sciences and Mathematics, Ural Federal University, Ekaterinburg 620002, Russia
}

\section{A R T I C L E I N F O}

\section{Keywords:}

ATP sulfurylase

Stanleya pinnata

Selenium

Hyperaccumulation

\begin{abstract}
A B S T R A C T
Background: The plant Stanleya pinnata hyperaccumulates Se up to $0.5 \%$ of its dry weight in organic forms, whereas the closely related Stanleya elata does not hyperaccumulate Se. ATP sulfurylase (ATPS) can catalyze the formation of adenosine 5'-phosphoselenate (APSe) from ATP and selenate. We investigated the $S$. pinnata ATPS2 isoform (SpATPS2) to assess its possible role in Se hyperaccumulation.

Methods: ATPS expression and activity was compared in the two Stanleya species. The ATPS2 protein sequences were modeled. Sub-cellular locations were analyzed using GFP fusions. Enzyme activity of purified recombinant SpATPS2 was measured.

Results: ATPS2 transcript levels were six-fold higher in roots of $S$. pinnata relative to $S$. elata. Overall root ATPS enzyme activity was two-fold elevated in $S$. pinnata. Cloning and sequencing of SpATPS2 and $S$. elata ATPS2 (SeATPS2) showed the predicted SeATPS2 to be canonical, while SpATPS2, although very similar in its core structure, has unique features, including an interrupted plastid targeting signal due to a stop codon in the $5^{\prime}$ region of the coding sequence. Indeed GFP fusions revealed that SpATPS2 had exclusive cytosolic localization, while SeATPS2 showed dual localization in plastids and cytosol. SpATPS2 activity was inhibited by both sulfate and selenate, indicating that the enzyme acts on both substrates.

Conclusions: The ATPS2 from S. pinnata differs from non-accumulator ATPS2 in its elevated expression and subcellular localization. It likely acts on both selente and sulfate substrates.

General significance: These observations shed new light on the role of ATPS2 in the evolution of Se hyperaccumulation in plants. This article is part of a Special Issue entitled Selenium research in biochemistry and biophysics - 200 year anniversary issue, edited by Dr. Elias Arnér and Dr. Regina Brigelius-Flohe.
\end{abstract}

\section{Introduction}

Sulfur is an essential element for all organisms [1]. Because sulfate is the dominant plant bioavailable form of sulfur in the environment, the sulfate assimilation pathway plays a critical role in plant growth and development $[2,3]$. ATP sulfurylase (ATPS, ATP: sulfate adenylyl transferase; EC 2.7.7.4) catalyzes the metabolic entry step into the sulfur assimilation pathway with the formation of adenosine-5'-phosphosulfate (APS) and inorganic pyrophosphate (PPi) from sulfate and
ATP. This reaction generates a phosphoric-sulfuric acid anhydride bond with high energy that helps to drive the sulfur assimilatory pathway [4-7]. Subsequent reduction of APS to sulfide and incorporation of sulfide into cysteine (Cys) brings a metabolic precursor for all compounds in organisms containing sulfur, including methionine, glutathione, iron-sulfur clusters, vitamin cofactors, as well as multiple secondary metabolites [8-11].

Genes encoding chloroplastic, mitochondrial, and cytosolic ATP sulfurylase isoforms have been cloned from potato [12], Indian mustard

\footnotetext{
This article is part of a Special Issue entitled Selenium research in biochemistry and biophysics - 200 year anniversary issue, edited by Dr. Elias Arnér and Dr. Regina Brigelius-Flohe.

* Corresponding author at: Department of Biology, 1878 campus delivery, Colorado State University, 200 West Lake Street, Fort Collins, CO 80523-1878, USA.

E-mail address: pilon@colostate.edu (M. Pilon).
} 
Table 1

Primers information used in this study.

\begin{tabular}{|c|c|c|c|}
\hline Oligonucleotides & $5^{\prime}-3^{\prime}$ sequences & Restriction site & Function \\
\hline SpinSela/ATPS1_Fw & CССTATCCTTTTGCT TCATCC & - & RT-PCR \\
\hline SpinSela/ATPS1_Re & GTGCTGCTTCATCCTCCAAC & - & \\
\hline SpinSela/ATPS2_Fw & CATCAAGAGGAACA TCATCAGC & - & \\
\hline SpinSela/ATPS2_Re & TTACAGGCTATCTCCAAAACAGC & - & \\
\hline SpinSela/ATPS4_Fw & GAGAAGGTGCTTGAG GATGG & - & \\
\hline SpinSela/ATPS4_Re & TTGGAGATGGGAAGATGGAG & - & \\
\hline SpinSela/Actin1_Fw & AGCATGAAGATCAAGG TGGTG & - & \\
\hline SpinSela/Actin1_Re & CTGACTCATCGTACTCTCCCT & - & \\
\hline Spin_Fw & GGAATTCCATATGCAATCTGTCACTTCCTCTT & NdeI & Plasmid construction for protein expression \\
\hline Spin_Rev & CGCGGGATCCTTAAGGCTTATCACTTTCTTGCA & BamHI & \\
\hline SpinSela_FwGFP & GAATGGTCGACATGTCTCTTCTGATCAGATCCT & Sall & Plasmid construction for subcellular localization \\
\hline Spin_ML_FwGFP & GAATGGTCGACCTGTCTCTTCTGATCAGATCCT & Sall & \\
\hline Spin_RevGFP & GCTCACCATGGCAGGCTTATCACTTTCTTGC & NcoI & \\
\hline Sela_RevGFP & CTCACCATGGCGGCTGAAACAACG & NcoI & \\
\hline
\end{tabular}

[13], and soybean [4]. Furthermore, four isoenzymes of ATP sulfurylase (ATPS1, - 2, -3, and -4) have been predicted in the Arabidopsis thaliana genome [14]. ATP sulfurylase enzyme activity was localized to two subcellular compartments in Arabidopsis: plastid and cytosol $[15,16]$. Rotte and Leustek [17] have suggested that cytosolic ATP sulfurylase plays a specialized function, e.g. to form sulfated metabolites and is less important for sulfate reduction. Recently, Arabidopsis ATPS2 was shown to encode for an ATPS enzyme with both plastidic and cytosolic localization, owing to multiple translation initiation sites [14]. Since chloroplasts are generally considered the primary site for sulfate assimilation, the chloroplastic isoform has been studied in detail. However, little is known about the specialized functions of the cytosolic isoform of ATPS in plants.

It is generally thought that selenate can be metabolized by $\mathrm{S}$ metabolic pathways, thus, after absorption, Se has the potential to be nonspecifically incorporated into any S-compound, because of the chemical similarity between Se and S [18-21]. Previous studies have documented that transgenic Brassica juncea which overexpress ATPS show increased (2.5-fold higher) shoot Se levels, increased selenate reduction to organic forms, and enhanced Se tolerance, when compared to wildtype [22,23]. Therefore, ATPS not only mediates selenate reduction in plants, but can also be rate limiting for selenate uptake and assimilation [22]. Transcriptome analyses showed that ATPS expression in root tissue of Arabidopsis was upregulated 6-fold by Se exposure [24].

Several members of the Brassicaceae family, such as Stanleya pinnata, take up extraordinarily high Se levels ( $>0.1 \%$ of dry weight) while growing in their native, seleniferous habitat, these are called Se hyperaccumulators [19]. They hyperaccumulate Se in all plant parts, predominantly in reduced form in organic compounds such as methylselenocysteine [19]. A comparison of the expression of ATPS gene family members between $S$. pinnata and Brassica juncea indicated that the hyperaccumulator has elevated expression levels of ATPS enzymes, particularly for ATPS2, which likely contributes to the capacity for the Se hyperaccumulation and hypertolerance [25]. In this study we asked the following questions: does a Se-specific ATPS2 exist in the hyperaccumulator $S$. pinnata? Are there different protein characteristics between ATPS2 for a Se hyperaccumulator and a non-hyperaccumulator? To date, no studies on the ATPS2 characterization of a Se hyperaccumulator have been described. To gain insight into the function of ATPS2 in the Se hyperaccumulator, first a gene expression study was performed via quantitative real-time reverse transcription polymerase chain reaction (qRT-PCR) to compare the differences in root gene expression levels between the Se hyperaccumulator species $S$. pinnata and the related non-accumulator $S$. elata grown with or without selenate. ATPS enzyme activity was also compared. The cDNAs encoding $S$. pinnata and $S$. elata ATPS were cloned, the sequences compared as well as modeled protein structures. In addition, protein subcellular localization was analyzed by constructing GFP fusions and transient expression in protoplasts. Finally, recombinant ATPS2 from $S$. pinnata was expressed in $E$. coli and purified, in order to investigate its relative sulfate/selenate specificity.

\section{Material and methods}

\subsection{ATP sulfurylase(ATPS) mRNA expression in the roots of $S$. pinnata and S. elata}

To evaluate the expression of three genes encoding members of the ATP sulfurylase family (ATPS1, ATPS2, ATPS4), qRT-PCR experiments were performed using a thermal cycler (Roche 480, Roche Diagnostics Corporation Indianapolis, IN) equipped with a 96 well plate system with the SYBR green PCR Master Mix reagent (Applied Biosystems, Thermo Fisher Scientific, Waltham, MA). For plants materials, the sources of both $S$. pinnata and $S$. elata seeds were described in our previous study [26], they were grown in a hydroponic system with $1 / 2$ strength Hoagland's solution for 2 weeks. Before harvest the plants were treated with or without $50 \mu \mathrm{M}$ Se as Na-selenate for $1 \mathrm{~h}$. after which time the plants were collected and separated into root and leaf samples, which were flash frozen for gene expression analysis. For each treatment, three biological replicates were used. RNA was extracted from roots of plants using a phenol/chloroform protocol described by Sambrook and Russell [27]. cDNA was prepared from DNase-treated total RNA prepared as described by El Mehdawi et al. [26]. Specific primer pairs for each of the relevant genes as well as the Actin 1 reference gene were designed for conserved sequences of Brassicaceae spp. (Table 1) and tested for their activity using annealing temperatures between 58 and $67^{\circ} \mathrm{C}$ by conventional PCR. The qPCR reactions were performed in a total volume of $10 \mu \mathrm{L}$, using serial dilutions of template cDNA in $1 \mu \mathrm{L}$, $1 \mu \mathrm{L}$ of each primer at $10 \mu \mathrm{M}$, and $5 \mu \mathrm{L}$ of $2 \times$ SYBR Green PCR Master Mix based on the instructions of manufacturer (Applied Biosystems, Thermo Fisher Scientific, Waltham, MA). All PCRs were performed as the following thermal cycling profile: $95^{\circ} \mathrm{C}$ for $10 \mathrm{~min}, 50$ cycles of $95^{\circ} \mathrm{C}$ for $15 \mathrm{~s}, 60^{\circ} \mathrm{C}$ for $1 \mathrm{~min}$. The analysis of expression of each sample was evaluated in two technical replicates and quantitative RT-PCR analyses were performed on three biological replicates. All quantifications were normalized to the Actin 1 housekeeping gene (Fig. 1). Expression values are given as the mean of the normalized expression values of the biological triplicates, calculated according to Eq. (2) of the Q-gene software [28].

\subsection{Cloning and plasmid construction}

Primers used for cloning are listed in Table 1 . The coding sequences for ATPS2 of S. pinnata and S. elata were amplified by PCR (using highfidelity Takara Taq polymerase, Takara Bio USA, Mountain view, CA) using root cDNA as a template. The cDNA was prepared from DNase- 

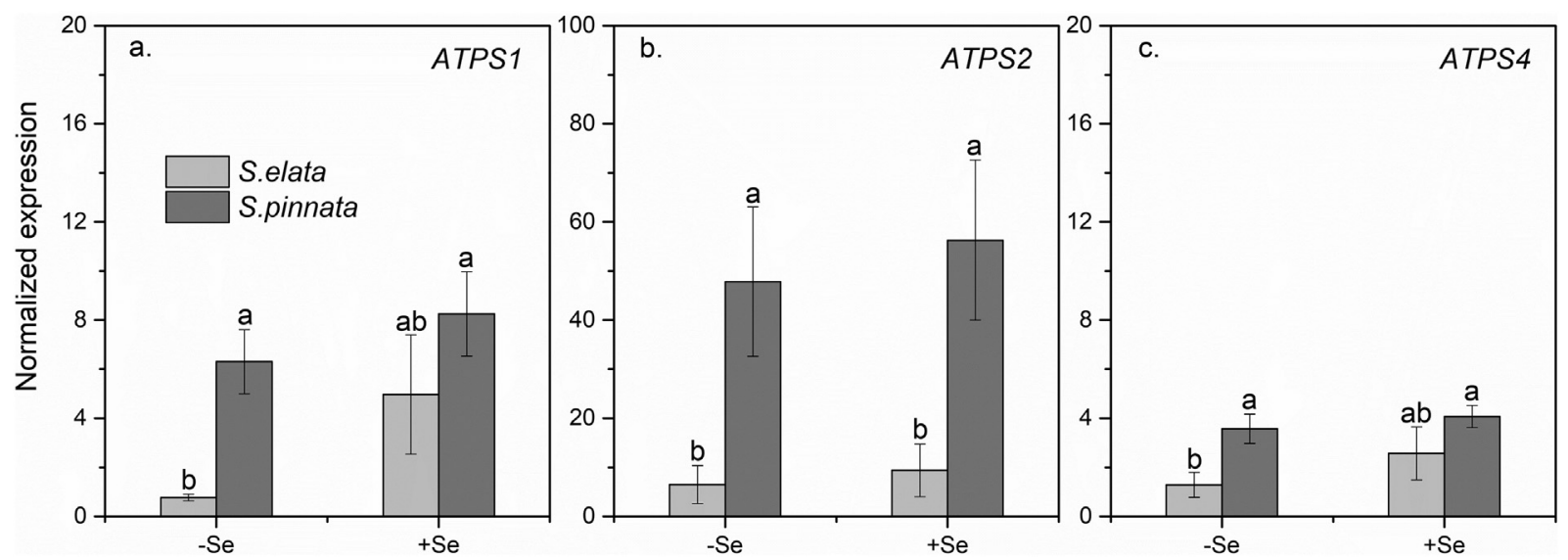

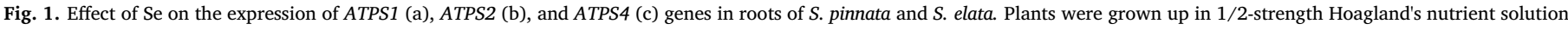

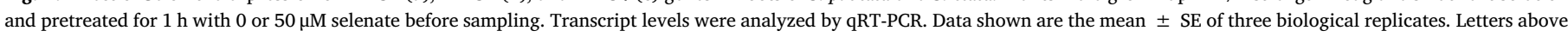
bars indicate statistically significant groups $(P<0.05)$.

treated total RNA prepared from 2-week old seedlings as described by Schiavon et al. [25]. For expression in bacteria, the mature sequence of S. pinnata ATPS2 was amplified using sets of primers with $\mathrm{NcoI}$ and BamHI restriction sites on the $5^{\prime}$ ends, the PCR product was digested with NcoI and BamHI and then ligated into vector pET28a (Novagen, Madison, WI) to produce plasmid pMATPS2 for expression as a 6Histagged protein (see supplemental Fig. S1).

For green fluorescent protein (GFP) localization, the GFP reporter plasmid $35 \Omega$-SGFP(S65T) [29] with the CaMV35S promoter sequence was used to generate ATPS2-GFP fusions. Due to the presence of a stop codon found in the transit peptide sequence for the ATPS2 in the $S$. pinnata species, we also made a construct in which the first start codon was mutated from ATG to CTG (encoding Leucine) via primer-mediated site-directed mutagenic PCR. For the GFP fusions, flanking SalI and $N c o$ I restriction sites were introduced via PCR. PCR products were digested with SalI and NcoI, ligated into the SalI-NcoI digested $35 \Omega$-SGFP (S65T) vector to generate full-length SeATPS2-GFP, SpATPS2-GFP as well as mutated version called SpATPS2-GFP ${ }^{\mathrm{M}-\mathrm{L}}$, respectively.

\subsection{Protein expression and purification}

For overexpression of SpATPS2, E. coli Rosetta (DE3) was transformed with pET28a-SpATPS2. Cells were grown at $37^{\circ} \mathrm{C}$ in LuriaBertani (LB) medium containing kanamycin $\left(50 \mu \mathrm{gL}^{-1}\right)$ until the culture reached an $A 600 \mathrm{~nm}$ of 0.8 . Two liters of LB medium containing $50 \mathrm{mg} \mathrm{L}^{-1}$ kanamycin was inoculated with 1:100 volume of overnight culture. Cells were grown at $37^{\circ} \mathrm{C}$ to an $A 600$ of 0.5 , and expression was induced by the addition of $0.4 \mathrm{mM}$ isopropyl- $\beta$-D- thiogalactopyranoside as described [30] followed by incubation for $3 \mathrm{~h}$ at $37^{\circ} \mathrm{C}$. The culture was chilled on ice, and the cells were collected by centrifugation for $5 \mathrm{~min}$ at $5000 \times g$ at $4^{\circ} \mathrm{C}$. From here on, all procedures were performed at $4{ }^{\circ} \mathrm{C}$ except where mentioned. The bacterial pellet was washed with $150 \mathrm{mM} \mathrm{NaCl}$ and resuspended in $50 \mathrm{mM}$ Tris-HCl, $\mathrm{pH} 7.5$, and then sonicated 5 times for $30 \mathrm{~s}$ with $1 \mathrm{~min}$ ice-cooling between sonication to disrupt the cells. The 6 His-tagged and cleaved SpATPS2 were purified essentially as described for CpSufE [30]. The lysate was centrifuged for $10 \mathrm{~min}$ at $1 \times 10^{6} \times \mathrm{g}$, and the cleared supernatant was loaded at a flow rate of $3 \mathrm{~mL} \mathrm{~min}{ }^{-1}$ onto His-Bind iminodiacetic acid (IDA)-agarose (Novagen, Madison, WI) in a $1.6 \times 20 \mathrm{~cm}$ column, which had been saturated with $200 \mathrm{mM} \mathrm{NiSO}_{4}$, washed, and equilibrated in $50 \mathrm{mM}$ Tris-HCl, pH 7.5. The column was washed with 4 volumes of $50 \mathrm{mM}$ Tris-HCl, pH 7.5, followed by 4 volumes of $1 \mathrm{M} \mathrm{NaCl}, 50 \mathrm{mM}$ Tris-HCl, pH 7.5, 6 volumes of $50 \mathrm{mM}$ Tris- $\mathrm{HCl}, \mathrm{pH} 7.5$, again, and 2 volumes of $0.1 \mathrm{M}$ imidazole in $50 \mathrm{mM}$ Tris- $\mathrm{HCl}, \mathrm{pH} 7.5$, respectively. 6His-APTS2 was eluted with 4 volumes of $1 \mathrm{M}$ imidazole in $50 \mathrm{mM}$ Tris$\mathrm{HCl}, \mathrm{pH} 7.5$. Fractions of $10 \mathrm{~mL}$ were collected and protein content measured according to Bradford [31]. Peak fractions were pooled and dialyzed overnight against $25 \mathrm{mM}$ Tris-HCl, $\mathrm{pH}$ 7.5. Pure 6His-ATPS2 ran as a single band on SDS-PAGE after staining with Coomassie Brilliant Blue. To produce cleaved ATPS2, the pooled peak fractions were dialyzed overnight against $20 \mathrm{mM}$ Tris-HCl, $\mathrm{pH} 8.4$, followed by incubation with thrombin in a 1:1,00 $(\mathrm{w} / \mathrm{w})$ ratio (thrombin/target protein) at $4{ }^{\circ} \mathrm{C}$ for $8 \mathrm{~h}$ in $20 \mathrm{mM}$ Tris- $\mathrm{HCl}, \mathrm{pH} 8.4,150 \mathrm{mM} \mathrm{NaCl}, 2.5 \mathrm{mM}$ $\mathrm{CaCl}_{2}$, as suggested by the manufacturer (Novagen, Madison, WI). The purified cleaved SpATPS2 was dialyzed overnight against $25 \mathrm{mM}$ Tris$\mathrm{HCl}, \mathrm{pH}$ 7.5. Purity was assessed by SDS-PAGE. Purified protein was flash-frozen in liquid nitrogen and stored at $-80^{\circ} \mathrm{C}$. Protein concentration was determined by the Bradford method (Protein Assay, BioRad, Hercules, CA) with bovine serum albumin (BSA) as standard [31].

\subsection{Enzyme assays of ATP sulfurylase}

Shoot and root samples for ATP sulfurylase enzyme analysis were collected from 3-w-old seedlings of $S$. pinnata and $S$. elata plants grown under hydroponic conditions with 1/2-strength Hoagland nutrient solution. The samples were collected and separated into shoots and roots, and immediately frozen, before grinding in liquid nitrogen. The frozen powder was then extracted with $1 \mathrm{~mL} \mathrm{~g}^{-1}$ fresh weight of a buffer containing $50 \mathrm{~mm}$ Tris, pH 8.0, 20\% glycerol, $2 \mathrm{mM}$ EDTA, and $0.1 \mathrm{mM}$ phenyl methane sulfonyl fluoride (PMSF). ATP sulfurylase enzyme activity was assayed in the reverse reaction, according to the method of Renosto et al. [32]. For ATP synthesis, the assays contained $50 \mathrm{mM}$ Tris$\mathrm{HCl}$ (pH 8.0), $5 \mathrm{mM} \mathrm{MgCl}_{2}, 0.3 \mathrm{mM} \mathrm{NADP}{ }^{+}, 1 \mathrm{mM}$ glucose, $1 \mathrm{mM} \mathrm{PPi}$, $10 \mathrm{U}$ of hexokinase (Sigma-Aldrich, St Louis, MO, and $5 \mathrm{U}$ of glucose-6phosphate dehydrogenase (Sigma-Aldrich) in $150 \mu \mathrm{L}$ reaction system. To evaluate the difference in inhibition on purified ATPS2 from $S$. pinnata between selenate and sulfate, the inhibitory assays contained $0.2 \mathrm{M}$ Na-selenate or Na-sulfate, or $0.2 \mathrm{M} \mathrm{NaCl}$, in $50 \mathrm{mM}$ Tris- $\mathrm{HCl}$ (pH 8.0), $5 \mathrm{mM} \mathrm{MgCl}_{2}, 0.3 \mathrm{mM} \mathrm{NADP}^{+}, 1 \mathrm{mM}$ glucose, $1 \mathrm{mM} \mathrm{PPi}, 10 \mathrm{U}$ of hexokinase, and $5 \mathrm{U}$ of glucose-6-phosphate dehydrogenase in $150 \mu \mathrm{L}$ reaction system. All reactions were initiated by addition $1.5 \mu \mathrm{L}$ of $11 \mathrm{mM}$ (final concentration $0.11 \mathrm{mM}$ ) adenosine-5'-phosphosulfate (APS). The change in absorbance at $340 \mathrm{~nm}$ was recorded and corrected for the background rate before adding APS. The extinction coefficient for absorbance of pyridine nucleotide at $340 \mathrm{~nm}$ of $6.22 \mathrm{mmol}^{-1} \mathrm{~cm}^{-1}$ was used to calculate the ATP sulfurylase activity.

\subsection{Sequence analysis and alignments}

Sequence alignment and analysis were performed using the multiple alignment program for amino acid or nucleotide sequences (MAFFT version 7, online version) and ExPASY translate tools (available online 


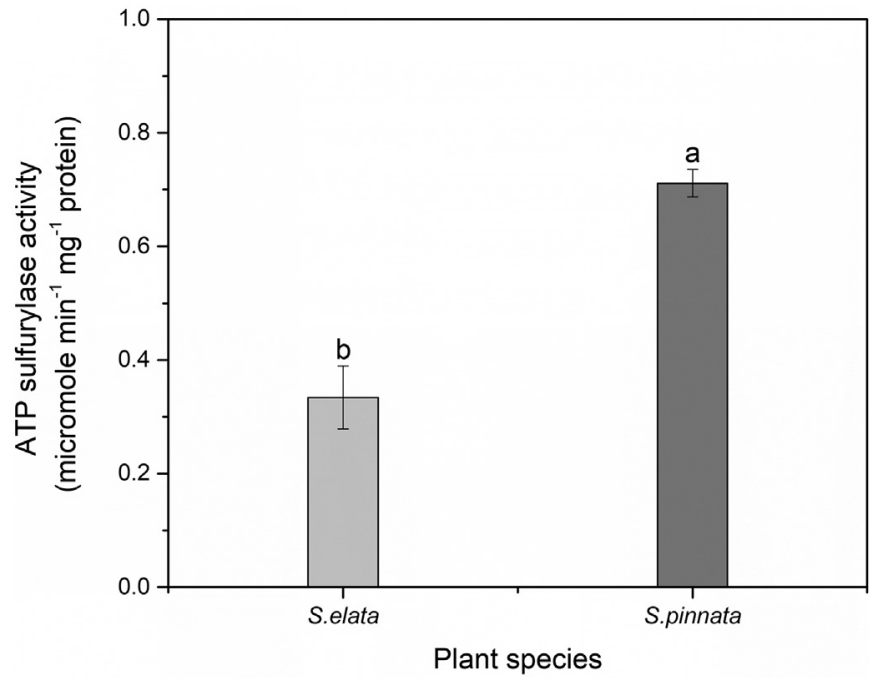

Fig. 2. ATP-sulfurylase activity in roots of 3 week-old plants of $S$. pinnata and S. elata. Values shown are the mean and SE of three samples. Letters above bars indicate significant differences between the means $(P<0.05)$.

at http://web.expasy.org/translate/). Searches for sequence similarity and characteristics were performed using the BLAST (Basic Local Alignment Search Tool) and CDD (Conserved Domains) network service provided by the National Center for Biotechnology Information (NCBI, available online at www.ncbi.nlm.nih.gov).

\subsection{Subcellular localization of GFP fusion proteins}

The plasmids encoding ATPS2-GFP fusions were transformed into Arabidopsis protoplasts, and expressed proteins were observed under a confocal microscope, as described by Abdel-Ghany et al. [33] with modification. GFP fusions were expressed in Arabidopsis protoplastderived cells from the $35 \mathrm{~S}$ promoter in the GFP reporter plasmid $35 \mathrm{~S} \Omega$ GFP(S65 T). For protoplast preparation, 5-week-old Arabidopsis plants (ecotype Columbia) were used. Fresh leaf tissue $(5 \mathrm{~g})$ was placed in $10 \mathrm{~mL}$ of a buffer containing $1.5 \%(\mathrm{w} / \mathrm{v})$ cellulase onozuka R-10 (RPI, Mt Prospect, IL), 0.4\% (w/v) macerozyme R-10 (RPI), 0.1\% (w/v) BSA, $10 \mathrm{mM} \mathrm{CaCl}_{2}, 20 \mathrm{mM} \mathrm{KCl}, 0.4 \mathrm{M}$ mannitol, $20 \mathrm{mM}$ MES, pH 5.7, vacuum infiltrated for $1 \mathrm{~min}$, and incubated over night at room temperature in dark condition. The clear digest was filtered through a 75$\mathrm{mm}$ nylon mesh, and the protoplasts were harvested by centrifugation for $2 \mathrm{~min}$ at $200 \times \mathrm{g}$ and washed twice in $20 \mathrm{~mL}$ of cold W5 wash solution (154 mM NaCl, $125 \mathrm{mM} \mathrm{CaCl}_{2}, 5 \mathrm{mM} \mathrm{KCl}, 2 \mathrm{mM}$ MES, pH 5.7). The pellet was suspended in $2 \mathrm{~mL}$ of mannitol/magnesium solution (15 $\mathrm{mM} \mathrm{MgCl}_{2}, 0.4 \mathrm{M}$ mannitol, $4 \mathrm{mM}$ MES, $\mathrm{pH}$ 5.7). Protoplasts were counted using a hemocytometer, and their concentration was adjusted to $4 \times 10^{6}$ intact cells $\mathrm{mL}^{-1}$ with mannitol/magnesium solution. One microgram of plasmid DNA, and $110 \mu \mathrm{L}$ of polyethylene glycol (PEG) solution (40\% PEG 4000, 0.2 M mannitol, $0.1 \mathrm{M} \mathrm{CaCl}_{2}$ ) were added to $100 \mu \mathrm{L}$ of the protoplast solution, very gently mixed, and allowed to incubate for $30 \mathrm{~min}$ at room temperature. The solution was very slowly diluted with $440 \mu \mathrm{L}$ of W5 solution and then pelleted by centrifugation for $2 \mathrm{~min}$ at $200 \times \mathrm{g}$. Protoplasts were resuspended in $1 \mathrm{~mL}$ of W5 solution, and incubated at room temperature for $16 \mathrm{~h}$ under continuous light. Fluorescence of ATPS2-GFP fusion proteins in protoplasts was obtained using an Olympus Fluoview 300 inverted confocal laserscanning microscope (Olympus, Waltham, MA), and images were captured by the Fluoview software and saved as Tiff files.

\subsection{Modeling and refinement of ATP sulfurylase2 from S. pinnata and $S$.} elata

The initial structure of ATPS2 was generated by a fully automated protein structure homology-modeling server [34] (SWISS-MODEL, available online at https://swissmodel.expasy.org) by using the structure of ATPS from soybean [35] as a search template. PyMOL (PyMOL Molecular Graphics System, Version 2.0.6 Schrodinger, LLC) was used for the further refinement of structure [36].

\subsection{Data statistical analysis}

The Statistical Analysis Software (SAS 9.0 Institute, USA) was used for statistical data analysis, the Duncan's multiple rank test was used to compare means of traits at $P=0.05$. All datasets were tested for normal distribution and equal variance.

\section{Results}

\subsection{Expression of ATPS and ATP sulfurylase activity}

To obtain insight into the expression levels of ATPS isoforms and the effects of the presence of Se, the transcript abundance was investigated in the roots of $S$. pinnata and $S$. elata plants using qRT-PCR (Fig. 1). Generally, the transcript levels of ATPS isoforms in roots showed a large difference between $S$. pinnata and $S$. elata: the transcripts for ATPS1, ATPS2 and ATPS4 were much more abundant in the Se hyperaccumulator than in the non-accumulator. Among these, a distinctly higher transcript level was observed for ATPS2 in the Se hyperaccumulator compared with ATPS1 and ATPS4 (Fig. 1, note scale difference). The expression of ATPS1 and ATPS4 isoforms tended to be enhanced by short-term selenate exposure in the Se non-accumulator, but, interestingly, this was not observed for the ATPS2 isoform. For the Se hyperaccumulator, selenate did not affect the transcript abundance of any of the three ATPS isoforms (Fig. 1). To determine the total ATP-sulfurylase enzyme activity in root tissue of both species, crude extracts from root tissue of 3-week old plants were used to perform an ATPS enzyme assay using the reverse enzyme reaction, which is most commonly used, as it is energetically favored. The Se hyperaccumulator showed over 2-fold higher ATP sulfurylase activity in the root compared with the Se non-accumulator (Fig. 2).

\subsection{Sequence alignment of ATPS2s and their sequence characteristics}

The full coding sequences of ATPS2 from $S$. pinnata and $S$. elata were cloned from root cDNA. Sequence analysis of the clones from $S$. pinnata and $S$. elata showed the presence of open reading frames encoding polypeptides of 480 and 488 amino acid residues, respectively. The predicted amino acid sequences were aligned with the published Arabidopsis lyrata sequence as shown in Fig. 3. Similarity analysis indicated that ATPS2 of $S$. pinnata and $S$. elata have $96 \%$ sequence similarity, whereas ATPS2 of $S$. pinnata has $93 \%$ sequence similarity with A. lyrata. ATPS2 proteins contain N-terminal extensions predicted to serve as transit peptides for chloroplast targeting [14]. However, interestingly, a stop codon was found in the transit peptide of $S$. pinnata (Fig. 3, red X). In addition, the hyperaccumulator ATPS2 showed a distinctly different $\mathrm{C}$ terminus than the two non-accumulator species, and 11 unique amino acid residues across its sequence (Fig. 3, highlighted in red).

\subsection{Structures of ATPS2 from S. pinnata and S. elata}

In order to see if the Stanleya ATPS2 proteins contain all features of active ATPS enzymes we did structural modeling of the predicted mature proteins. The modeled 3D structures of ATPS2 from S. pinnata (A) and S. elata (B) are shown in Fig. 4, and the detailed helix and strand 

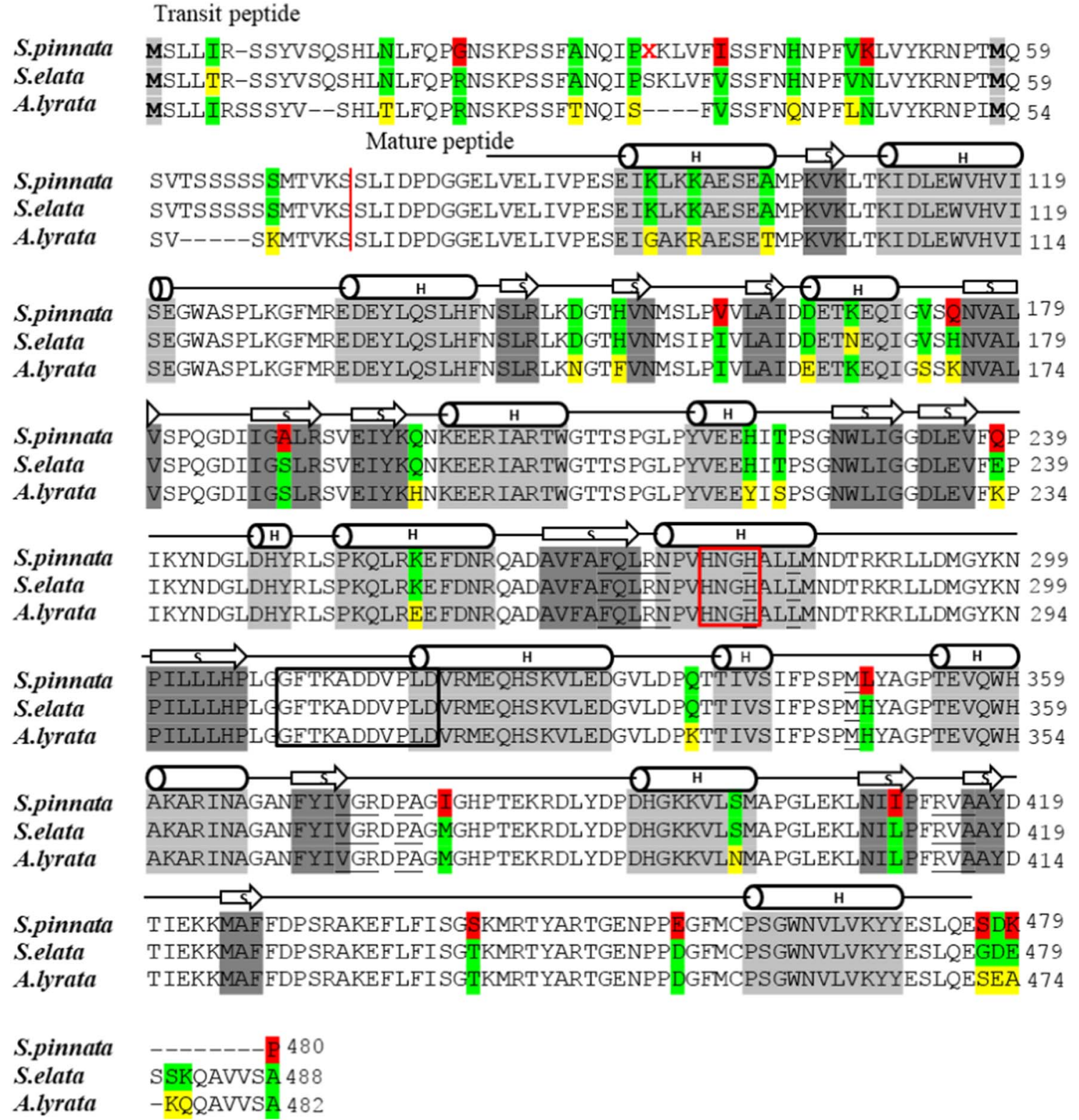

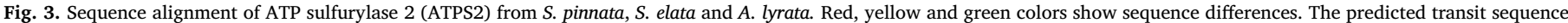

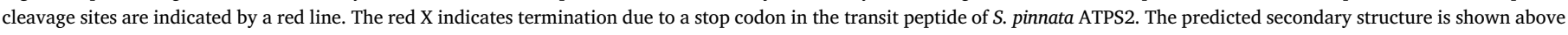

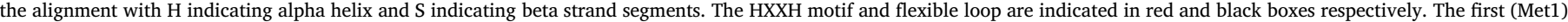

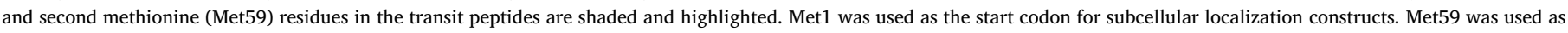
start codon for protein expression cloning. The predicted residues of the active site are underlined.

structure are labeled above the amino acid sequence alignment in Fig. 3. We used the Met59 of the precursor sequences (see Fig. 3) as a start point of the mature proteins. Thus, from here on, all amino acid numbering starts at this Met except where mentioned. It should be noted however that the N-termini and C-termini of the Stanleya ATPS2 sequences could not be modeled due to lack of overlap with template models in the database. Thus the 3D models in Fig. 4 start with the sequence LTEPDG and end with KYYESL, corresponding to the end of the last alpha helix indicated in Fig. 3. ATPS proteins are characterized by the presence of several conserved amino acid sequences, most notably a conserved HXXH motif at the active site and a flexible loop (GXTKADDVPLD) close to the active site [37,38]. The sequence alignment (Fig. 3) and structure modeling (Fig. 4) showed a conserved HNGH motif located in a helix that is part of the active site, as well as the loop sequence (GFTKADDVPLD) in all three species. The analysis of conserved regions indicates that ATPS2 from $S$. pinnata and $S$. elata contains a PUA-like domain belonging to the PUA_2 super family, which is generally found at the N-terminus of ATP sulfurylase enzymes [36]. Another feature of ATPS2 from S. pinnata and $S$. elata is that a flexible loop (residues 241-250) is positioned over the active site, as mentioned above (Fig. 3). This mobile loop may be in a "closed" or "down" as well as in an "open" or "up" position corresponding with occupancy of the active site, as reported for the crystal structures of microbial ATP sulfurylases $[38,39]$. With the prediction of the enzyme active site, both model building and conserved domain analysis indicate the same amino acid residues within the SpATPS2 and SeATPS2 active sites (Fig. 4; for detailed views see Supplementary Figs. S2 and S2). The unique amino acid sequences found in ATPS2 from $S$. pinnata, relative to non-accumulators S. elata and A. lyrata (Fig. 3, red highlights) might alter oligomeric interactions between the chains to some extent, but were not present in the conserved regions of ATPS2 necessary for catalysis (Fig. 4). 


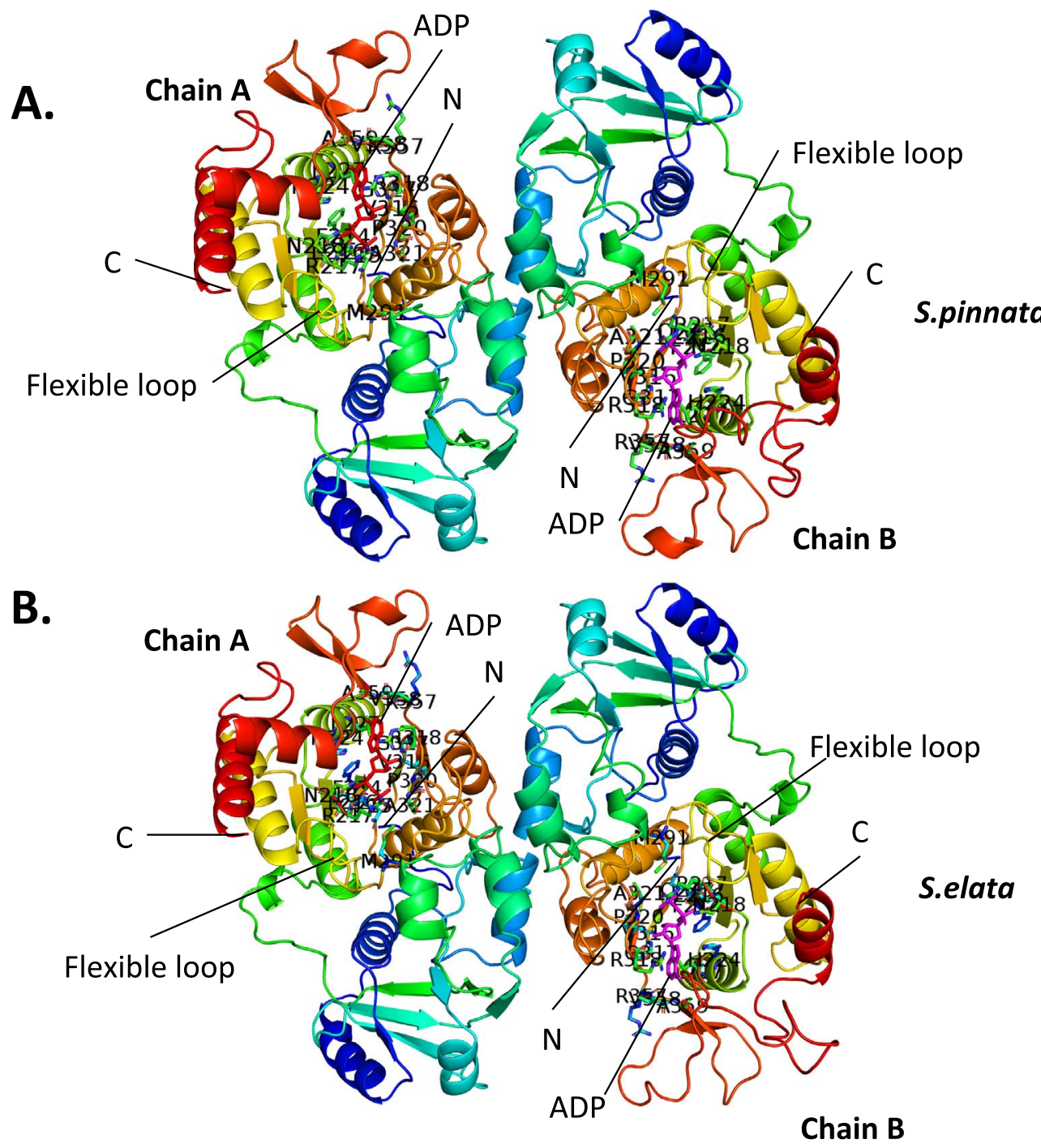

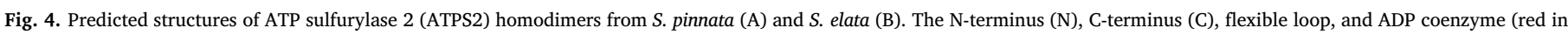

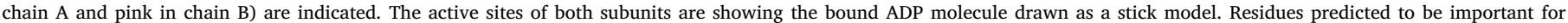

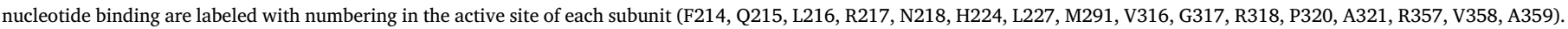

\subsection{Subcellular localization of ATPS2 from S. pinnata and S. elata}

To determine the subcellular localizations of ATPS2 from S. pinnata and $S$. elata, the full-length sequences of ATPS2 from $S$. pinnata and $S$. elata were fused to GFP. In addition, we constructed a mutant version of the fusion for ATPS2 from $S$. pinnata with Met $^{1}$ converted to Leu ${ }^{1}$ so that translation should initiate at the Met close to the predicted start of the mature sequence (Met58 in Fig. 3). This mutant was designed to investigate the possible effect of a stop codon in the transit peptide on the expression and subcellular localization of ATPS2 from $S$. pinnata. The resultant ATPS2-GFP constructs were transformed and expressed in Arabidopsis protoplasts (Fig. 5). Both $S$. pinnata ATPS2-GFP fusions, the "native" (SpATPS2-GFP) and the mutated ATPS2 (SpATPS2-GFP ${ }^{\mathrm{M}-\mathrm{L}}$ ) were expressed but they only showed localization to the cytosol and not in the plastids, as evidenced by the lack of overlapping fluorescence with the red chlorophyll auto-fluorescence signals (Fig. 5 A and B). However, in the transfection with the GFP fusion constructs for ATPS2 of $S$. elata (SeATPS2-GFP), GFP was observed with both cytosolic and chloroplastic localization, as indicated by the overlay of green fluorescence and red auto-fluorescence from chlorophyll (Fig. 5; panels $\mathrm{C}$ and D show two different cells transfected with the same construct). The extent of cytosolic location seemed to depend on relative expression. These observations suggest that ATPS2 of $S$. elata can have dual localization in the plant, similar to what was reported before for Arabidopsis [14]. Importantly, the stop codon in the S. pinnata transit sequence did not abolish expression of SpATPS2-GFP. We conclude that for both SpATPS2-GFP and SpATPS2-GFP ${ }^{\mathrm{M}-\mathrm{L}}$ translation must have started at the AUG close to the start of the predicted mature sequence, which appears to have changed the localization of ATPS2 to exclusively cytosolic in this Se hyperaccumulator species.

\subsection{Effect of $S$ and se on enzyme activity of purified S. pinnata ATPS2}

ATPS2 from S. pinnata (SpATPS2) was overexpressed in E. coli as an $\mathrm{N}$-terminally His-tagged protein and purified using $\mathrm{Ni}^{2+}$-affinity chromatography, as described in the Methods section; then the His-tag was cleaved with thrombin to obtain the purified protein. ATPS is predicted to be a homodimer, according to the structure modeling. The SDS-PAGE and immunoblot gel analysis indicated that the subunit molecular weight of the purified SpATPS2 was $\sim 48 \mathrm{kDa}$, which is the expected size (Fig. 6).

Because it is energetically favored in vitro, the reverse ATPS reaction is commonly assayed, which measures the formation of ATP and sulfate from APS (present at $0.11 \mathrm{mM}$ ) and PPi. To investigate the 


\section{A. SpATPS2_GFP}

B. SpATPS2_GFP M_L

C. SeATPS2_GFP

\section{SeATPS2_GFP}

Bright field

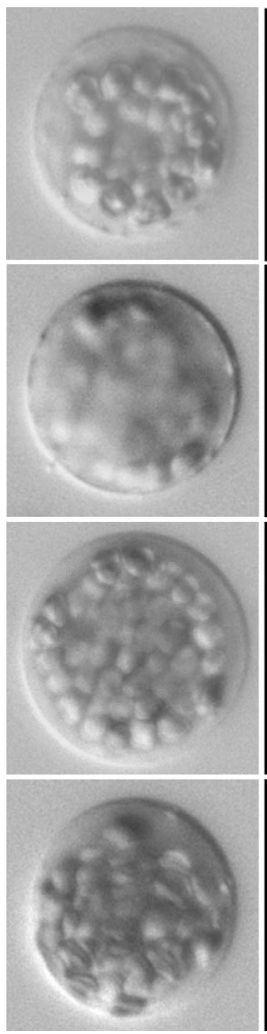

GFP
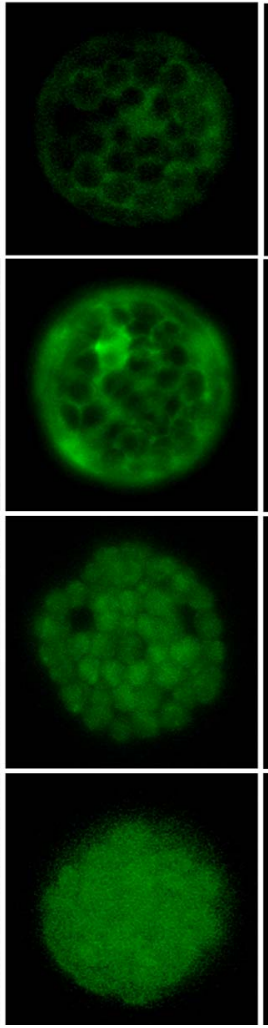

Chlorophyll
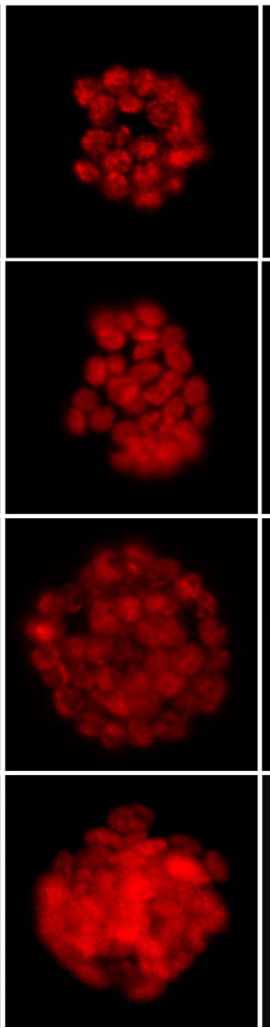

Overlay

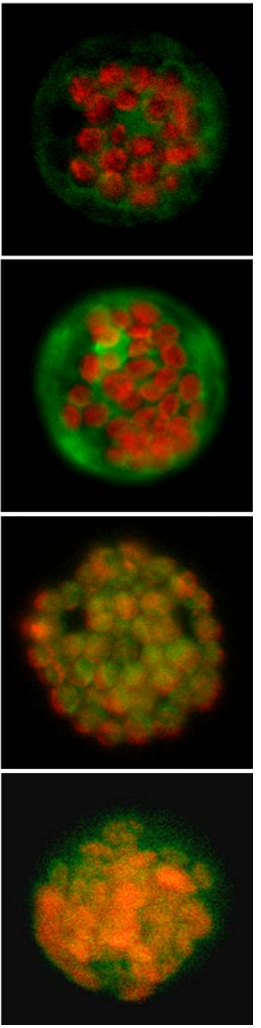

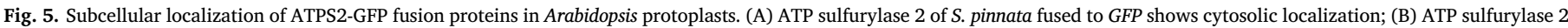

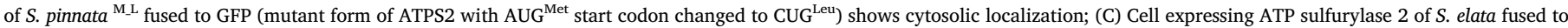

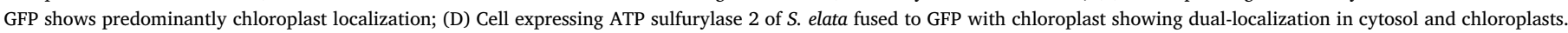
Bright-field, GFP fluorescence (green), chlorophyll fluorescence (red), and merged images (green and red) are shown.

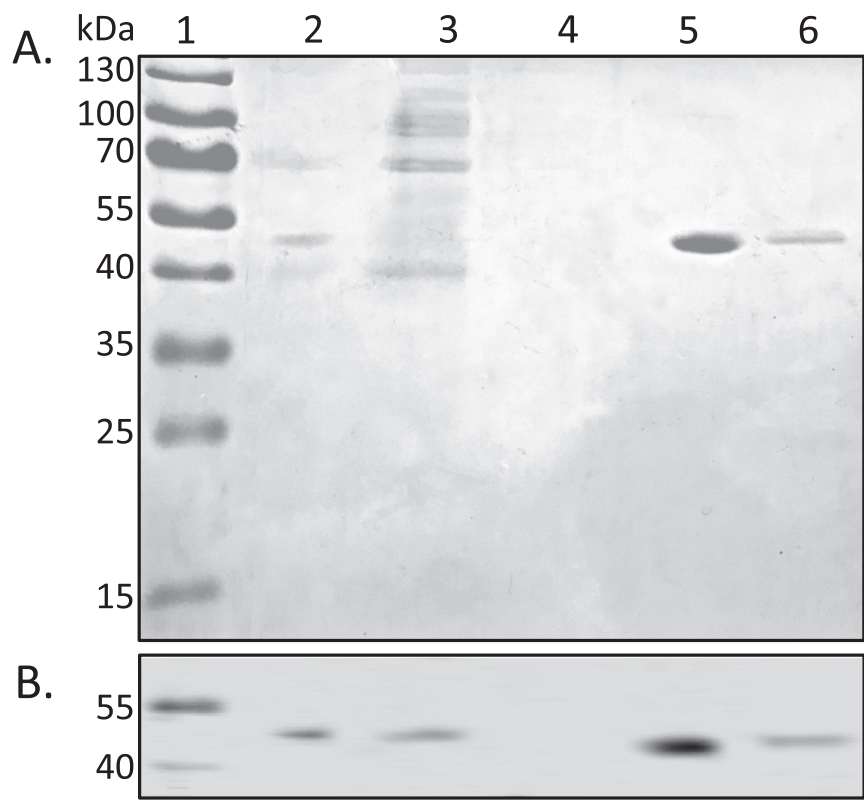

Fig. 6. Purification of ATPS2 from S. pinnata (A) SDS-PAGE (12.5\% gel) stained with Coomassie Brilliant Blue. (B) immunoblot analysis using his-tag antibody. Lane 1, molecular mass standards. Lane 2: The flow through of Ni-NTA column (lane 2),Lane $3+4$ : $50 \mathrm{mM}$ Tris $\mathrm{pH} 7.5,1 \mathrm{M} \mathrm{NaCl}$ wash fractions. Lanes $5+6$ eluate in $1 \mathrm{M}$ imidazole. $2 \mu \mathrm{g}$ of protein was loaded.

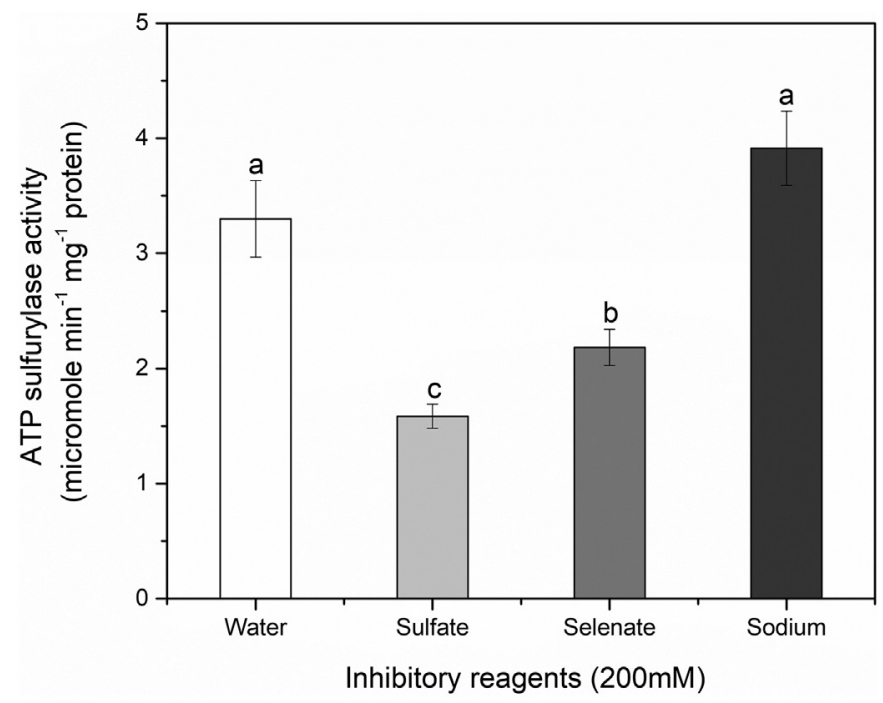

Fig. 7. Effects of sulfate, selenate or $\mathrm{NaCl}$ on enzyme activity of purified ATP-sulfurylase 2 from $S$. pinnata. Salts were added to $200 \mathrm{mM}$. Water was used in the control. Values shown are the average and SE of three samples. Letters above bars indicate significant groups $(P<0.05)$.

sulfate/selenate substrate specificity of purified SpATPS2, we analyzed the inhibitory effect of $0.2 \mathrm{M}$ sodium sulfate or sodium selenate on this reverse enzyme activity. As controls, either the same volume of $\mathrm{H}_{2} \mathrm{O}$ was used, or $0.2 \mathrm{M}$ sodium chloride to control for any salt effect. We expected that addition of excess sulfate should partly inhibit ATP formation (driving the reaction back towards APS and PPi formation). We 
were interested to test if selenate could also affect the reaction, possibly revealing a preference of the enzyme for either the $S$ or Se substrate. The activity of purified SpATPS2 was significantly $(\sim 50 \%)$ inhibited by $200 \mathrm{mM}$ sodium sulfate, as well as by $200 \mathrm{mM}$ sodium selenate $(\sim 33 \%)$. As expected there was no significant effect of sodium chloride $(\mathrm{NaCl})$ on the reaction suggesting that the effects of sulfate and selenite were not just due to salt effects on the enzyme (Fig. 7). These findings indicate that SpATPS2 can also use selenate as a substrate, and does not prefer selenate over sulfate in the reaction as assayed. In fact, the enzyme was slightly more inhibited by sulfate than selenate (Fig. 7).

\section{Discussion}

This study sheds new light on the potential role of ATP sulfurylase in Se hyperaccumulation in $S$. pinnata. Compared to non-accumulator $S$. elata, the Se hyperaccumulator had 5-fold higher root transcript levels of ATP sulfurylase 2, and 2-fold higher root ATPS enzyme activity (collective activity from all four isoforms). Interestingly, the SpATPS2 contains a stop codon in its plastid targeting sequence, resulting in purely cytosolic localization, while ATPS2 of $S$. elata showed dual localization in plastids and cytosol, as also reported earlier for spinach and $A$. thaliana $[14,40]$. In addition to differences in its predicted transit sequence, SpATPS2 was uniquely different from its $S$. elata and A. lyrata homologues in its $C$ terminus and nine other amino acid residues, none of which are in the predicted active site or mobile loop. Inhibition by sulfate or selenate of in vitro reverse ATPS activity assays using purified recombinant SpATPS2 indicates that the enzyme can use selenate as a substrate, but does not show a preference for selenate over sulfate.

Based on these results, ATPS2 appears to be the most abundant isoform in the Se hyperaccumulator, while in non-accumulators ATPS1 is most abundant. The finding that the main ATPS activity in the hyperaccumulator appears to be exclusively cytosolic is intriguing because sulfate (and selenate) assimilation is generally thought to happen mostly in plastids. The cytosolic ATPS activity is considered to be more important for the synthesis of sulfated metabolites like glucosinolates, via the intermediate $3{ }^{\prime}$-phospho-5'-adenylylsulfate(PAPS) [41]. Based on the activity assays of the purified SpATPS2, this enzyme likely can utilize selenate as a substrate. This likely is a general property of ATP sulfurylases, judged from earlier studies where overexpression of another ATPS isoform (AtAPS1) in B. juncea enhanced Se assimilation, as well as accumulation and tolerance [22,23]. Considering the high transcript level of ATPS2 in S. pinnata compared to the same transcript in S. elata and to the transcript levels of other ATPS isoforms in both species, it is reasonable to hypothesize that $S$ and Se metabolic fluxes in the Se hyperaccumulator are altered relative to those in Se non-accumulators, particularly in view of the cytosolic location of the SpATPS2. This is of particular interest in the context of the plant's ability to hyperaccumulate Se. Perhaps the high cytosolic SpATPS2 levels enable the hyperaccumulator to quickly metabolize incoming selenate and sulfate, thereby reducing cytosolic selenate and sulfate concentrations and stimulating root sulfate transporter activities. Interesting to note in this respect is that Sultr1;2 has been found to be very much upregulated in $S$. pinnata [26]. Interesting is also the earlier observation that overexpression of AtATPS1 in B. juncea stimulated S and Se accumulation [22]. More studies are needed to elucidate the subsequent fate of the APSe produced in the cytosol of $S$. pinnata roots. It may either be converted in the cytosol via PAPSe to downstream selenated metabolites, or its Se may still find a way to be metabolized to methyl-selenocysteine, the main form of Se in this species [19]. All APR isoforms are reportedly plastidic in plants [42]. Further conversion from selenite to SeCys and methyl-SeCys probably readily proceeds. It is also possible, considering that the overexpression of SpATPS2 is mainly in the root [25], that high root cytosolic ATPS activity facilitates translocation of Se to the shoot, a known feature of this hyperaccumulator [26].

The specific activity of the purified ATPS2 protein was $3.5 \mu \mathrm{mol}$ substrate per minute per milligram protein in the ATP synthesis reaction, which is relatively low compared to the activity of earlier reported ATP sulfurylases [2,4]. It does not appear that the SpATPS2 is selenate-specific, based on the activity assays of the purified SpATPS2. Whole-plant selenate uptake studies have shown that selenate uptake by $S$. pinnata is only marginally inhibited by high sulfate, in contrast to $S$. elata or B. juncea [26]. The mechanism of this selenate specificity does not appear to involve selective activation in the root cells, based on the new results presented here, but rather may involve one or more selenate-specific transporters.

In conclusion, the findings reported here provide new insights into the metabolic mechanisms in Se hyperaccumulator plants. The high ATP sulfurylase 2 activity in the cytosol and concomitant reduced ATPS activity in the plastids of the root likely divert Se (and S) fluxes in the plant that in turn may lead to enhanced root selenate uptake and perhaps translocation and assimilation. The availability of the purified SpATPS2 enzyme opens the possibility to do further follow-up structural and kinetic studies to reveal the possible importance of its unique amino acids and better understand its role in Se metabolism in this hyperaccumulator.

\section{Transparency document}

The Transparency document associated this article can be found, in the online version.

\section{Acknowledgements}

This work was supported by National Science Foundation grant IOS1456361 to E.A.H.P-S, and the authors also thank the China Scholarship Council for providing a graduate research fellowship to Ying Jiang (grant No. 201606350049). Tripti is highly grateful to the Ministry of Education and Science of the Russian Federation (02. A03.21.0006) for providing a mobility fund fellowship.

\section{Author contributions}

YJ, EPS and MP designed and performed most of the experiments, analyzed the data and wrote the manuscript. MS, LWL RJ, and AEM helped with the cloning and purification of APS protein. RJ, LWL and Tripti helped with the enzyme assay and collected the data. SR performed the protoplast imaging, and $\mathrm{ZZ}$ and $\mathrm{YH}$ helped perform the computational analysis and editing of the manuscript. All authors read and approved the manuscript.

\section{Conflict of interest}

The authors declare that they have no conflict of interest.

\section{Appendix A. Supplementary data}

Supplementary data to this article can be found online at https:// doi.org/10.1016/j.bbagen.2018.03.014.

\section{References}

[1] T. Leustek, M.N. Martin, J.A. Bick, J.P. Davies, Pathways and regulation of sulfur metabolism revealed through molecular and genetic studies, Annu. Rev. Plant Physiol. Plant Mol. Biol. 51 (2000) 141-165.

[2] M. Murillo, T. Leustek, ATP sulfurylase from Arabidopsis thaliana and Escherichia coli are functionally equivalent but structurally and kinetically divergent. Nucleotide sequence of two ATP sulfurylase cDNAs from Arabidopsis thaliana and analysis of a recombinant enzyme, Arch. Biochem. Biophys. 323 (1995) 195-204.

[3] J.C. Davidian, S. Kopriva, Regulation of sulfate uptake and assimilation - the same or not the same? Mol. Plant 3 (2010) 314-325.

[4] P. Phartiyal, W.S. Kim, R.E. Cahoon, J.M. Jez, H.B. Krishnan, A.T.P. Soybean, Sulfurylase, a homodimeric enzyme involved in sulfur assimilation, is abundantly expressed in roots and induced by cold treatment, Arch. Biochem. Biophys. 450 (2006) 20-29.

[5] H. Takahashi, Regulation of sulfate transport and assimilation in plants, Int. Rev. 
Cell Mol. Biol. 281 (2010) 129-159.

[6] T. Leustek, K. Saito, Sulfate transport and assimilation in plants, Plant Physiol. 120 (1999) 637-643.

[7] G.E. Ravilious, J. Herrmann, L.S. Goo, C.S. Westfall, J.M. Jez, Kinetic mechanism of the dimeric ATP sulfurylase from plants, Biosci. Rep. 33 (2013) 585-591.

[8] R. Hell, H. Hillebrand, Plant concepts for mineral acquisition and allocation, Curr. Opin. Biotechnol. 12 (2001) 161-168.

[9] J.W. Mueller, N. Shafqat, Adenosine-50-phosphosulfateea multifaceted modulator of bifunctional 30-phospho-adenosine-50-phosphosulfate synthases and related enzymes, FEBS J. 280 (2013) 3050e3057.

[10] M. Sauter, Phytosulfokine peptide signalling, J. Exp. Bot. 66 (2015) 5161e5169.

[11] M. Klein, J. Papenbrock, The multi-protein family of Arabidopsis sulphotransferases and their relatives in other plant species, J. Exp. Bot. 55 (2004) 1809e1820.

[12] D. Klonus, R. Höfgen, L. Willmitzer, J.W. Riesmeier, Isolation and characterization of two cDNA clones encoding ATP-sulfurylases from potato by complementation of a yeast mutant, Plant J. 6 (1994) 105-112.

[13] S. Heiss, H. Schäfer, A. Haag-Kerwer, T. Rausch, Cloning sulfur assimilation genes of Brassica juncea L.: cadmium differentially affects the expression of a putative low affinity sulfate transporter and isoforms of ATP sulfurylase and APS reductase, Plant Mol. Biol. 39 (1999) 847-857.

[14] A.S. Bohrer, N. Yoshimoto, A. Sekiguchi, N. Rykulski, K. Saito, H. Takahashi, Alternative translational initiation of ATP sulfurylase underlying dual localization of sulfate assimilation pathways in plastids and cytosol in Arabidopsis thaliana, Front. Plant Sci. 5 (2015) 750.

[15] H.M. Logan, N. Cathala, N. Grignon, J.C. Davidian, Cloning of a cDNA encoded by a member of the Arabidopsis thaliana ATP sulfurylase multigene family: expression studies in yeast and in relation to plant sulfur nutrition, J. Biol. Chem. 271 (1996) 12227-12233.

[16] Y. Hatzfeld, S. Lee, M. Lee, T. Leustek, K. Saito, Functional characterization of a gene encoding a fourth ATP sulfurylase isoform from Arabidopsis thaliana, Gene 248 (2000) 51-58.

[17] C. Rotte, T. Leustek, Differential subcellular localization and expression of ATP sulfurylase and 5'-adenylylsulfate reductase during ontogenesis of Arabidopsis leaves indicates that cytosolic and plastid forms of ATP sulfurylase may have specialized functions, Plant Physiol. 124 (2000) 715-724.

[18] T.A. Brown, A. Shrift, Selenium: toxicity and tolerance in higher plants, Biol. Rev. Camb. Philos. Soc. 57 (1982) 59-84.

[19] M. Schiavon, E.A.H. Pilon-Smits, The fascinating facets of plant selenium accumulation-biochemistry, physiology, evolution and ecology, New Phytol. 213 (2017) 1582-1596.

[20] N. Terry, A.M. Zayed, M.P. de Souza, A.S. Tarun, Selenium in higher plants, Annu. Rev. Plant Physiol. 51 (2000) 401-432.

[21] Y.G. Zhu, E.A.H. Pilon-Smits, F.J. Zhao, P.N. Williams, A.A. Meharg, Selenium in higher plants: understanding mechanisms for biofortification and phytoremediation, Trends Plant Sci. 14 (2009) 436-442.

[22] E.A.H. Pilon-Smits, S.B. Hwang, C.M. Lytle, Y.L. Zhu, J.C. Tai, R.C. Bravo, Y.C. Chen, T. Leustek, N. Terry, Overexpression of ATP sulfurylase in Brassica juncea leads to increased selenate uptake, reduction and tolerance, Plant Physiol. 119 (1999) 123-132.

[23] T. Van Huysen, N. Terry, E.A.H. Pilon-Smits, Exploring the selenium phytoremediation potential of transgenic Brassica juncea overexpressing ATP sulfurylase or cystathionine- $\gamma$-synthase, Int. J. Phytorem. 6 (2004) 111-118.

[24] D. Van Hoewyk, H. Takahashi, A. Hess, M. Tamaoki, E.A.H. Pilon-Smits, Transcriptome and biochemical analyses give insights into selenium-stress responses and selenium tolerance mechanisms in Arabidopsis, Physiol. Plant 132 (2008) 236-253.

[25] M. Schiavon, M. Pilon, M. Malagoli, E.A.H. Pilon-Smits, Exploring the importance of sulfate transporters and ATP sulphurylases for selenium hyperaccumulation-a comparison of Stanleya pinnata and Brassica juncea (Brassicaceae), Front. Plant Sci. 6 (2015) 2.

[26] A.F. El Mehdawi, Y. Jiang, Z.S. Guignardi, A. Esmat, M. Pilon, E.A.H. Pilon-Smits, M. Schiavon, Influence of sulfate supply on selenium uptake dynamics and expression of sulfate/selenate transporters in selenium hyperaccumulator and nonhyperaccumulator Brassicaceae, New Phytol. 217 (2018) 194-205.

[27] J. Sambrook, D.W. Russell, Molecular Cloning: A Laboratory Manual, Vol. 1 Cold Spring Harbor Laboratory Press, New York, NY, 2001.

[28] P.Y. Muller, H. Janovjak, A.R. Miserez, Z. Dobbie, Processing of gene expression data generated by quantitative real-time RT-PCR, BioTechniques 32 (2002) $1372-1379$.

[29] S. Miras, D. Salvi, M. Ferro, D. Grunwald, J. Garin, J. Joyard, N. Rolland, Noncanonical transit peptide for import into the chloroplast, J. Biol. Chem. 277 (2002) 47770-47778.

[30] H. Ye, S.E. Abdel-Ghany, T.D. Anderson, E.A.H. Pilon-Smits, M. Pilon, CpSufE activates the cysteine desulfurase CpNifS for chloroplastic Fe-S cluster formation, J. Biol. Chem. 281 (2006) 8958-8969.

[31] M.M. Bradford, A rapid and sensitive method for the quantitation of microgram quantities of protein utilizing the principle of protein-dye binding, Anal. Biochem. 72 (1976) 248-254.

[32] F. Renosto, R.L. Martin, J.L. Borrel, D.C. Nelson, I.H. Segel, ATP sulfurylase from trophosome tissue of Riftia pachyptila (hydrothermal vent tube worm), Arch. Biochem. Biophys. 290 (1991) 66-78.

[33] S.E. Abdel-Ghany, J.L. Burkhead, K.A. Gogolin, N. Andrés-Colás, J.R. Bodecker, S. Puig, L. Peñarrubiab, M. Pilon, AtCCS is a functional homolog of the yeast copper chaperone Ccs1/Lys7, FEBS Lett. 579 (2005) 2307-2312.

[34] M. Biasini, S. Bienert, A. Waterhouse, K. Arnold, G. Studer, T. Schmidt, F. Kiefer, T.G. Cassarino, M. Bertoni, L. Bordoli, T. Schwede, SWISS-MODEL: modelling protein tertiary and quaternary structure using evolutionary information, Nucleic Acids Res. 42 (2014) W252.

[35] J. Herrmann, G.E. Ravilious, S.E. McKinney, C.S. Westfall, S.G. Lee, P. Baraniecka, M. Giovannetti, S. Kopriva, H.B. Krishnan, J.M. Jez, Structure and mechanism of soybean ATP sulfurylase and the committed step in plant sulfur assimilation, J. Biol. Chem. 289 (2014) 10919-10929.

[36] K. Parey, U. Demmer, E. Warkentin, A. Wynen, U. Ermler, C. Dahl, Structural, biochemical and genetic characterization of dissimilatory ATP sulfurylase from Allochromatium vinosum, PLoS One 8 (2013) e74707.

[37] V. Saridakis, D. Christendat, M.S. Kimberi, A. Dharamsi, A.M. Edwards, E.F. Pai, Insights into ligand binding and catalysis of a central step in $\mathrm{NAD}^{+}$synthesis: structures of Methanobacterium thermoautotrophicum NMN adenyltransferase com plexes, J. Biol. Chem. 276 (2001) 7225-7232.

[38] J.D. Beynon, I.J. Macrae, S.L. Huston, D.C. Nelson, I.H. Segel, A.J. Fisher, Crystal structure of ATP sulfurylase from the bacterial symbiont of the hydrothermal vent tubeworm Riftia pachyptila, Biochemistry 40 (2001) 14509-14517.

[39] T.C. Ullrich, R. Huber, The complex structures of ATP sulfurylase with thiosulfate, $\mathrm{ADP}$ and chlorate reveal new insights in inhibitory effects and the catalytic cycle, J. Mol. Biol. 313 (2001) 1117-1125.

[40] F. Renosto, H.C. Patel, R.L. Martin, C. Thomassian, G. Zimmer-Man, I.H. Segel, ATP sulfurylase from higher plants: kinetic and structural characterization of the chloroplast and cytosol enzymes from spinach leaf, Arch. Biochem. Biophys. 307 (1993) 272-285.

[41] H. Chen, B. Zhang, L.M. Hicks, L. Xiong, A nucleotide metabolite controls stress responsive gene expression and plant development, PLoS One 6 (2011) e26661.

[42] S. Kopriva, A. Koprivova, Plant adenosine 5'-phosphosulphate reductase: the past, the present, and the future, J. Exp. Bot. 55 (2004) 1775-1783. 